\title{
THE VLBA CALIBRATOR SURVEY
}

\author{
A.J. BEASLEY, V. DHAWAN, E.B. FOMALONT, R.C. WALKER \\ AND J.M. WROBEL \\ NRAO, Socorro, New Mexico 87801-0387, USA
}

\section{Phase-referencing}

Using phase-referencing, the coherent integration time of VLBI observations can be substantially increased, permitting observations of weaker ( $\sim \mathrm{mJy})$ target sources (see e.g. Beasley \& Conway 1995). The position of a source can also be accurately measured relative to a reference source, allowing absolute and proper-motion measurements, optical-radio image alignment, and alignment of images made at different frequencies.

To make phase-referencing routinely available to the VLBI community, a suitable grid of phase-reference calibrators is needed. We have begun a VLBA survey of $\sim 3000$ flat-spectrum radio sources, selected from the Jodrell Bank-VLA Astrometric Survey (JVAS; Patnaik et al. 1992), in order to derive an "all-sky" catalog of phase-reference calibrators (see Figure 1). When complete, all JVAS sources detected above $100 \mathrm{mJy}$ at $8.4 \mathrm{GHz}$ on VLA A-array scales will be observed with the VLBA S/X dual-frequency system, using a geodetic-style frequency setup of four IFs at $2.3 \mathrm{GHz}$ spanning $100 \mathrm{MHz}$ and four IFs at $8.4 \mathrm{GHz}$ spanning $400 \mathrm{MHz}$. Each source is observed at three separated hour-angles for $100 \mathrm{~s}$, i.e. a total of $300 \mathrm{~s}$. Approximately $25 \%$ of the observing time is spent observing geodeticgrade calibrators. After amplitude and delay calibration in the NRAO AIPS software package, fringe solutions are transferred to GSFC CALC/SOLVE geodetic package to derive source positions. The calibrated data are also automatically imaged using AIPS and the Caltech Difmap package.

\section{Scientific Impact}

Our sample is statistically complete, and will be the largest set of sources surveyed with VLBI to date. Potential scientific returns from this survey include: (1) the detection of compact gravitational lenses, probing the existence of massive compact lensing objects such as $10^{6} \mathrm{M}_{\odot}$ black holes; (2) 
structural information and the brightness temperature distribution at 8.4 and $2.3 \mathrm{GHz}$ for a large sample of AGNs; (3) in combination with optical identifications and redshifts, this structural information will contribute to $\theta-\mathrm{z}$ and $\mu-\mathrm{z}$ studies.

\section{Current Status}

Three of fourteen epochs have been observed to date (11/95), a total of 648 sources in the declination ranges $0-22^{\circ}$ and $50-79^{\circ}$. The mean positional accuracy achieved is $\sim 0.7-1$ mas rms, sufficient for most phase-referencing purposes. Images from the survey data have typically a $2-3 \mathrm{mJy} \mathrm{rms}$, with a 100:1 dynamic range or better. At present, the plan is to complete all JVAS sources above $200 \mathrm{mJy}$ during 1996, with the entire survey complete by late 1997 . The survey positions, images and UV-data will be available online from the NRAO WWW server from early 1996. Special requests to survey selected regions to lower flux densities near important targets will be considered.

\section{VLBA Calibrator Survey Sources -- Observed(crosses), Planned(points)}

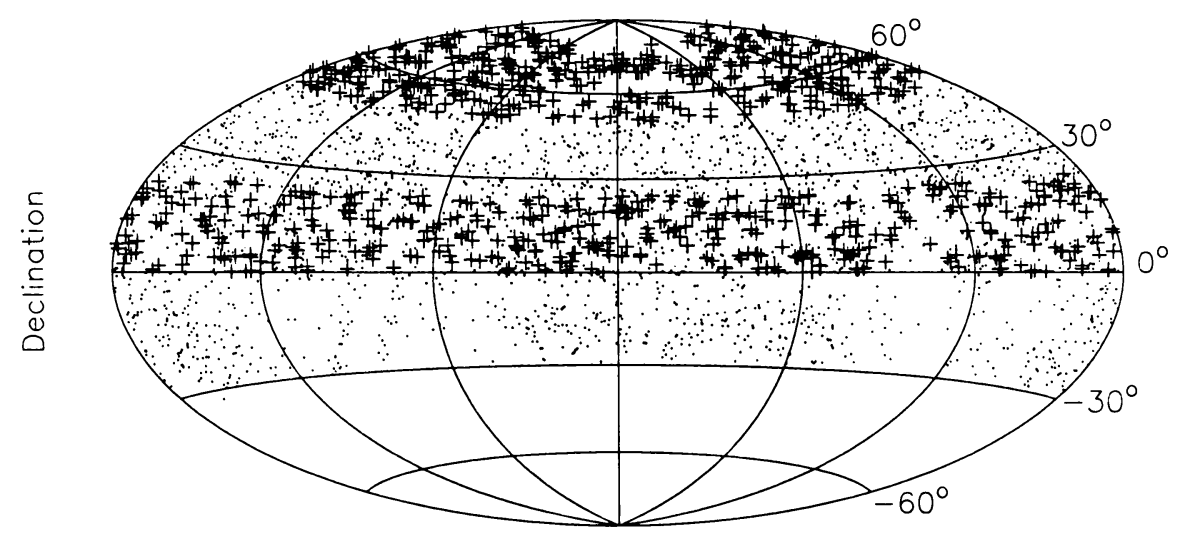

Right Ascension

Figure 1.

\section{References}

Beasley, A.J. \& Conway, J.E. 1995, in VLBI Phase-Referencing, Chapter 7, Proceedings of the NRAO VLBA Summer School, ASP Conference Series Vol. 82, eds. Zensus, A., Diamond, P.J. and Napier, P.J..

Patnaik, A.R. et al. 1992, MNRAS, 254, 655. 